\title{
Where have all the mentors gone?
}

\author{
Kevin J. Koomalsingh, MD, and Nahush A. Mokadam, MD
}

\author{
From the Division of Cardiothoracic Surgery, Department of Surgery, University of Washington, Seattle, Wash. \\ Disclosures: N.A.M. is a consultant to Abbott and Medtronic and an investigator for Abbott, Medtronic, and Syn- \\ Cardia. K.J.K. has nothing to disclose with regard to commercial support. \\ Received for publication Dec 20, 2017; accepted for publication Dec 27, 2017; available ahead of print Feb 2, \\ 2018. \\ Address for reprints: Nahush A. Mokadam, MD, Division of Cardiothoracic Surgery, Department of Surgery, Uni- \\ versity of Washington, 1959 NE Pacific St, Box 356310, Seattle, WA 98195 (E-mail: mokadamn@uw.edu). \\ J Thorac Cardiovasc Surg 2018;155:2057 \\ $0022-5223 / \$ 36.00$ \\ Copyright (C) 2018 by The American Association for Thoracic Surgery \\ https://doi.org/10.1016/j.jtcvs.2017.12.091
}

The robust surgeon-scientist culture, which once prevailed in cardiothoracic surgical training, has rapidly dwindled to mediocrity and is potentially hovering on extinction. It is a sobering reality that merits awareness and restitution. Present day surgeons are facing higher than ever pressures that preclude research endeavors. Increasing clinical scrutiny, administrative demands, diminished funding opportunities, and the work-life balance have all contributed to the fading surgeon-scientist. ${ }^{1}$ Nevertheless, recapturing and rebuilding this tradition is imperative to preserving the future generation of academic cardiothoracic surgeons.

In this month's edition of the Journal, Narahari and colleagues $^{2}$ present their findings shedding a favorable light on residents supported by T32 training grants. We applaud the authors for drawing attention to a vital component of cardiothoracic surgical training. In their study, they compared throughput at 2 academic institutions, both with rich histories of T32 training grants, versus 2 similar sized academic institutions without the same dedicated support structure. The results were quite impressive. Trainees supported by a T32 training grant were more likely to pursue cardiothoracic surgery after residency, publish more articles, obtain subsequent National Institutes of Health funding, and pursue advanced fellowships. Perhaps most surprising from this study is the underuse of the T32 training grant by cardiothoracic training programs. With just 2 programs maintaining a consistent track record, much work remains. Nevertheless, the grant continues to be an integral funding source for multiple training programs across academic medicine. In 2017, there were more than 2500 awards at 25 academic centers in the country. ${ }^{3}$

The message is clear-we need to reenergize our training programs with dedicated, structured, mentored research time to restore our surgeon-scientist culture. Residents should be encouraged to embrace a 2- to 3-year hiatus for protected research endeavors. Interest should be sparked as early as possible. Project ideas should align with a specific mentor, either internal or external, and manifest into a formal research proposal with prespecified goals. Ideally, if this can be arranged before the culmination of the second year of training, the ability to capitalize on the

\section{References} December 12, 2017. December 12, 2017.

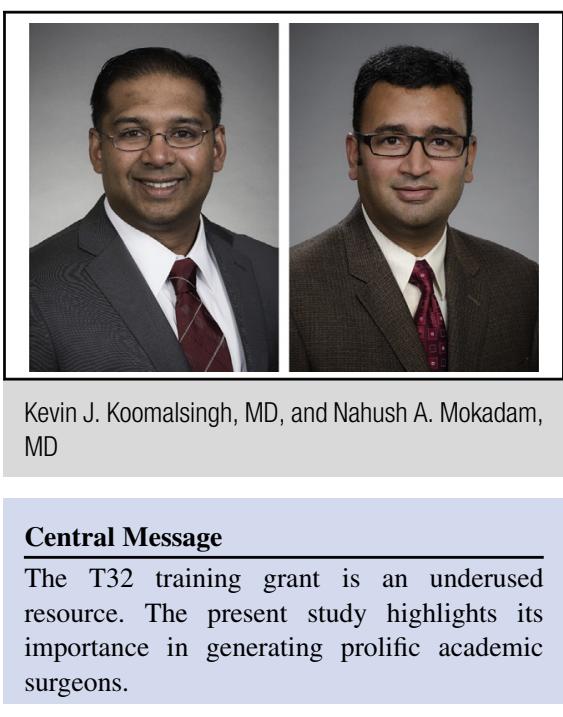

See Article page 2050.

T32 training grant may be achieved from both an economic and a regulatory perspective. Having National Institutes of Health-caliber checks and balance will ensure the program's utmost quality and integrity. ${ }^{4}$ Once all essential elements are in place, academic reproducibility, in line with Narahari and colleagues, ${ }^{2}$ may be observed. The net effect can be infectious. Chief residents will be emulated and continually inspire their junior counterparts. Mentors will be responsible for maintaining this momentum. And the recipients of these training grants can provide the basis for future mentorship.

Although the fate of our academic heritage is questioned, we are adequately poised for recourse. Mentors must rise above the challenges and reclaim their roles as surgeon-scientists. We need to cultivate inquisition and exploit effective funding mechanisms. Narahari and colleagues $^{2}$ demonstrated incredible academic value with the T32 training grant. It would behoove us to capitalize on this opportunity.

1. Keswani SG, Moles CM, Morowitz M, Zeh H, Kuo JS, Levine MH, et al. The future of basic science in academic surgery: identifying barriers to success for surgeon-scientists. Ann Surg. 2017;265:1053-9.

2. Narahari AK, Charles EJ, Mehaffey JH, Hawkins RB, Schubert SA, Tribble CG, et al. Cardiothoracic surgery training grants provide protected research time vital to the development of academic surgeons. J Thorac Cardiovasc Surg. 2018;155:2050-6.

3. T32 Training Grant. Available at: https://www.grantome.com. Accessed

4. Ruth L. Kirschstein Institutional National Research Service Award. Available at: https://researchtraining.nih.gov/programs/training-grants/T32. Accessed 